\section{The magnetic resonance revolution in brain imaging: impact on neonatal intensive care}

\section{N J Robertson, J S Wyatt}

Magnetic resonance imaging and spectroscopy techniques have huge medical and scientific potential in neonatal brain imaging

$\mathrm{T}$ he magnetic resonance (MR) phenomenon was first described in $1946^{12}$ but it was not until 1981 that the first transverse image through the human head was reported. ${ }^{3}$ In the last 20 years, MR technology has revolutionised medical and scientific neuroimaging, providing the richest source of information about the living brain available from any imaging technology and without the use of ionising radiation. MR techniques most commonly used in the neonate have been conventional MR imaging and ${ }^{31} \mathrm{P}$ and ${ }^{1} \mathrm{H} \mathrm{MR}$ spectroscopy. MR imaging has allowed the observation of brain development and response to perinatal brain injury in vivo with an unprecedented sensitivity for assessment of changes in grey and white matter and an ability to differentiate unmyelinated from myelinated white matter. MR spectroscopy has provided a metabolic fingerprint of the brain during normal development and after perinatal brain injury (fig 1). Conventional MR imaging and MR spectroscopy, however, are only part of an array of methods that comprise the MR diagnostic armamentarium, which includes diffusion weighted MR imaging, diffusion tensor imaging, MR angiography, functional MR imaging (fMRI), magnetisation transfer imaging, and chemical shift imaging. These newer techniques have huge medical and scientific potential.

Abbreviations: $\mathrm{CT}$, computed tomography FMRI, functional MR imaging; $\mathrm{HI}$, hypoxiaischaemia; $M R$, magnetic resonance; $N E$, neonatal encephalopathy; PVL, periventricular leucomalacia; US, ultrasonography; WMD, white matter damage
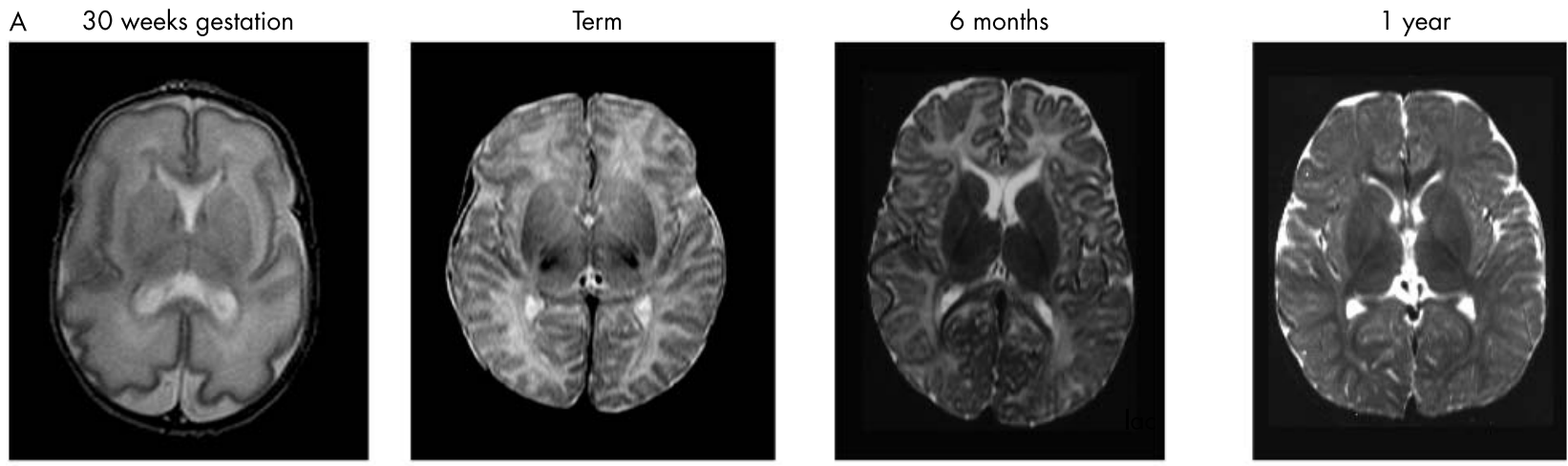

B
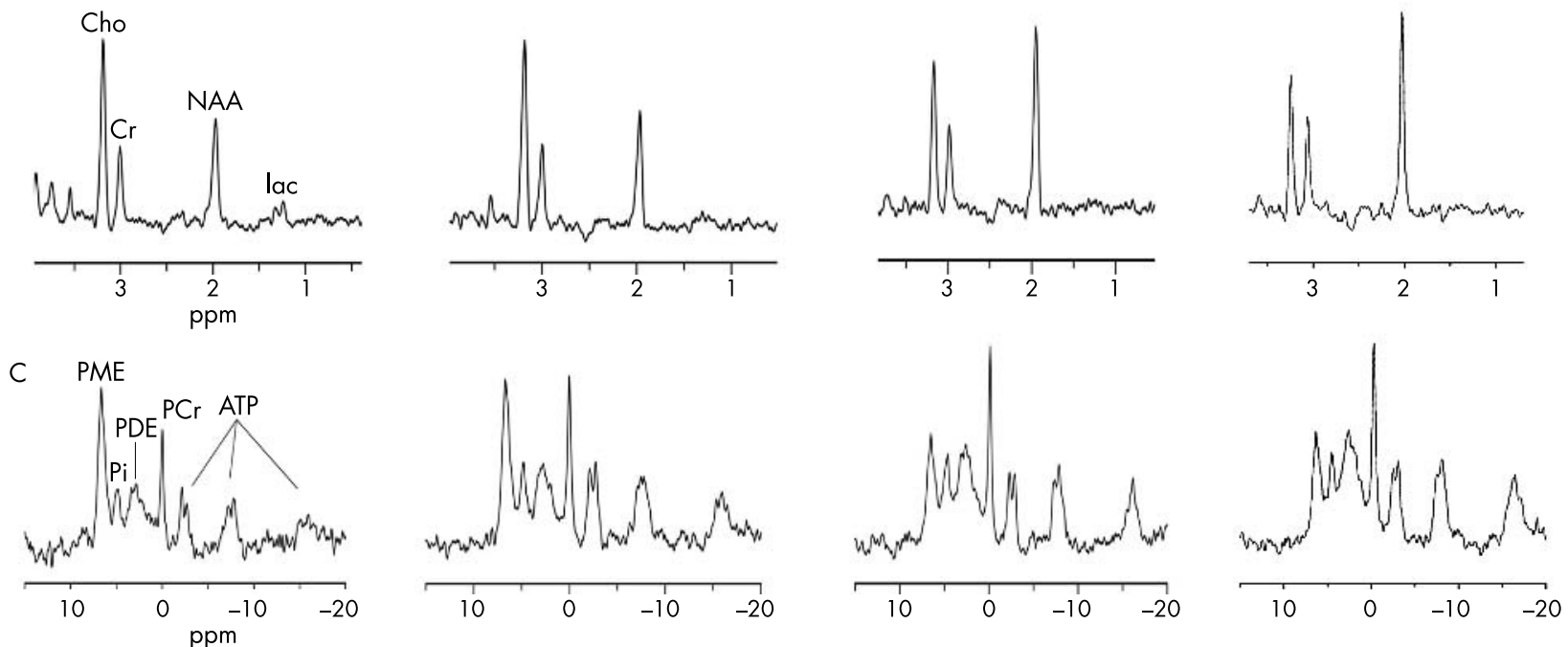

Figure 1 (A) Representative T2 weighted magnetic resonance (MR) images, (B) ${ }^{1} H$ MR spectra, and (C) ${ }^{31} P$ MR spectra from healthy infants at 30 weeks gestation, term, 6 months, and 1 year of age. (A) The MR images show an increase in volume, surface area, and sulcation of cerebral cortex and in volume and microstructural organisation of cerebral white matter with development. (B) The series of ${ }^{1} \mathrm{H} M R$ spectra show a steady increase in $\mathrm{N}$-acetyl aspartate (NAA; a marker of neuronal and axonal density and viability) and a decrease in brain lactate with maturation. (C) The series of ${ }^{31} \mathrm{P}$ $M R$ spectra show changing ratios of brain phospholipids and increasing energy state with maturation. $\mathrm{PME}$, Phosphomonoesters; $\mathrm{Pi}$, inorganic phosphate; PDE, phosphodiesters; PCr, phosphocreatine; ATP, adenosine triphosphate; Cho, choline; $\mathrm{Cr}$, creatine; lac, lactate. 


\section{RATIONALE FOR THE USE OF MR IN THE NEONATAL BRAIN}

In the last 20 years, we have seen major advances in obstetric and neonatal intensive care such as the introduction of new treatments and evolving modes of ventilatory support. Despite these advances, perinatal hypoxia-ischaemia (HI) is experienced by about 4/1000 live term births, and disability rates among very low birthweight (birth weight $<1500 \mathrm{~g})$ infants remain high, with $5-15 \%$ showing major spastic abnormalities and an additional $25-50 \%$ or more having deficits in cognition and behaviour. ${ }^{4-6}$ Neuroimaging is needed in at risk infants, as clinical evaluation may not provide adequate diagnostic or prognostic information and has two principal functions: (a) early diagnosis of brain injury so that appropriate medical management can be provided; (b) detection of lesions associated with long term neurodevelopmental disability. Currently, cranial ultrasonography (US), computed tomography (CT), and MR imaging are the most widely used techniques. MR imaging has a unique power as a diagnostic neuroimaging tool in neonatology.

\section{MR IMAGING IN NEONATOLOGY: BEST PRACTICE RECOMMENDATIONS Neonatal encephalopathy (NE)}

In the encephalopathic term infant, a conventional MR image provides the most specific means to predict the pattern of neuromotor outcome. The pattern of tissue injury is related to the type and severity of insult, and a close

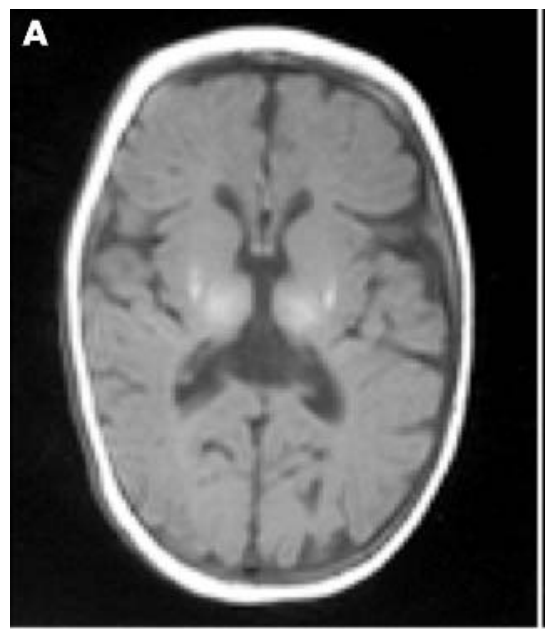

correlation exists between the pattern of abnormality on MR images and neurodevelopmental outcome. ${ }^{78}$ The most predictive finding on MR for abnormal outcome is a change in the signal intensity of the posterior limb of the internal capsule, ${ }^{8}$ and detailed descriptions of precise MR findings and their relation to later neurological outcome are available. ${ }^{9}$ In particular, MR imaging has refined the differentiation between global and focal injury in which the outcome is significantly different (fig 2). In addition, MR imaging enables the diagnosis of a range of cerebral developmental abnormalities, which may be confused with acute perinatal HI. MR imaging and spectroscopy also aid in the diagnosis of congenital metabolic disorders, which may present with acute NE. MR is particularly good at visua-

lising posterior fossa structures and diagnosing cerebellar malformations compared with US. ${ }^{10}$

US has the advantage of being mobile and easily used on the neonatal unit. It is therefore ideal for sequential scans to follow the evolution of brain lesions, defining normal cerebral anatomy, detecting large established basal ganglia lesions (from 1-3 days after birth), ${ }^{11}$ abnormal cerebral blood flow velocity (from 24 hours from birth), ${ }^{12}$ and major intracerebral haemorrhage. However, US has very limited accuracy in defining the site and extent of ischaemic tissue injury after perinatal HI. CT involves exposure to a considerable amount of radiation, making it unsuitable for serial scans. However, it is an effective

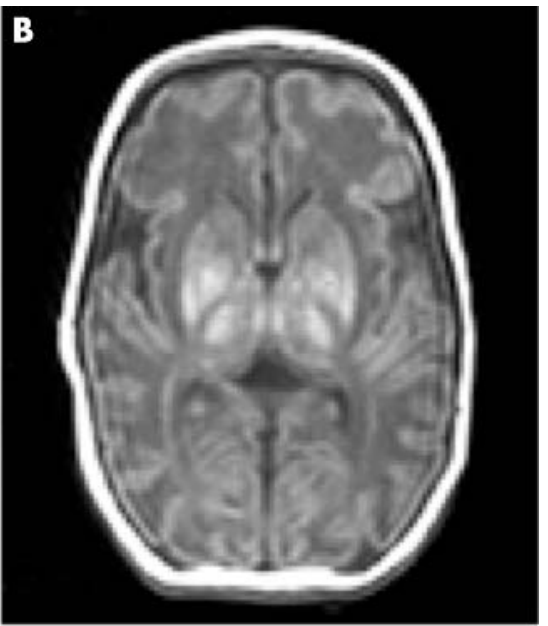

Figure 2 T1 weighted magnetic resonance images of $(A)$ focal injury in an infant with Sarnat stage II neonatal encephalopathy (NE) aged 11 days and (B) global injury in an infant with Sarnat stage III NE aged 12 days. (A) Focal high signal intensity lesions are visible in the lentiform nuclei and thalami, and there is loss of the normal high signal intensity from myelin in the posterior limb of the internal capsule. (B) There are extensive high signal intensity lesions in the lentiform nuclei and thalami, loss of the normal high signal intensity from myelin in the posterior limb of the internal capsule, and abnormal low signal intensity in the white matter with loss of the normal grey/white matter differentiation. technique for the detection of intracranial haemorrhage and may detect some basal ganglia and thalamic lesions.

In our opinion, there is now sufficient evidence to recommend that a conventional MR image should be performed to provide precise predictive information in all term infants with moderate or severe NE. A similar recommendation has been made recently by the American Academy of Neurology quality standards committee and endorsed by the American Academy of Pediatrics in 2002. ${ }^{13}$ The optimal timing appears to be toward the end of the first week after birth (and certainly within the neonatal period before atrophy becomes obvious). Very early scans performed in the first 24 hours can be misleading, as they may appear almost normal even when there is severe HI injury.

It is unlikely that MR will have a role in screening infants for entry into trials of neuroprotective interventions such as hypothermia. As the therapeutic window for neuroprotective treatments appears to be less than six hours, a conventional MR image will not give prognostic information within this time frame before the onset of the cascade of irreversible injury. So far, diffusion weighted imaging has not been proven to be effective as a prognostic tool in NE. ${ }^{14}$ This is surprising, as diffusion weighted imaging in the adult brain ${ }^{15}$ and experimental models ${ }^{16}$ of stroke has revolutionised the possibility of early diagnosis and intervention, often revealing image abnormalities on water diffusion maps well before CT or conventional $\mathrm{MR} .{ }^{31} \mathrm{P}$ and ${ }^{1} \mathrm{H}$ MR spectroscopy (giving quantitative information on brain energetics, intracellular $\mathrm{pH}$ $\left(\mathrm{pH}_{\mathrm{i}}\right)$ and lactate) can give early prognostic information on brain injury severity. However, for logistical reasons it is almost impossible to transport sick infants with NE to an MR system and perform an MR study within six hours of birth. The optimal method for selecting infants for neuroprotective trials of hypothermia is amplitude integrated electroencephalography (cerebral function monitoring), which has a high predictive value for neurological impairment in most, ${ }^{17}$ but not all, ${ }^{18}$ situations when performed as early as three hours after birth.

\section{Neonatal cerebral infarction}

As a consequence of the wider availability of MR brain imaging in the newborn infant, neonatal cerebral infarction has become increasingly recognised; cerebral arterial infarction occurs in about 1 in 4000 term infants who typically do not show signs of birth asphyxia, but present with seizures in the first few hours or days of life. The 
cause is usually unknown, although there is an association with inherited or acquired prothrombotic disorders. ${ }^{19}$ Early MR imaging is more sensitive than US in the detection of arterial infarcts, ${ }^{20}$ and diffusion weighted imaging is valuable in early grading of the lesion. ${ }^{21}$ MR imaging has allowed a more accurate prognosis to be assigned to infants with arterial infarction compared with US and CT. Generally only about $20 \%$ of infants develop a hemiplegia after neonatal cerebral arterial infarction; the likelihood of motor impairment depends on the overall extent of injury and involvement of cerebral hemispheres, basal ganglia, and posterior limb of the internal capsule and the background activity on neonatal electroencephalography. ${ }^{22}$ Wherever possible, MR imaging and cerebral function monitoring should become a standard investigation in infants with early neonatal seizures.

\section{Preterm brain injury}

Epidemiological studies have confirmed that the main correlate of major long term neurological deficits in the preterm infant is periventricular white matter damage (WMD). ${ }^{23}$ WMD encompasses at least three main groups: cystic periventricular leucomalacia (PVL), haemorrhagic parenchymal infarction, and diffuse WMD. US is the most appropriate safe and accessible bedside tool for assessing brain structure, intraventricular haemorrhage, germinal layer haemorrhage, haemorrhagic parenchymal infarction, cystic PVL, and ventriculomegaly. Sequential scans are now standard in the neonatal unit for monitoring evolving brain injury. Such frequent sequential scans are necessary to diagnose cystic PVL; an area of echodensity appears 24-48 hours after a known insult, but cysts are not visible on US for a further two to four weeks.

US has limitations in the diagnosis of subtle preterm WMD. Abnormal echogenicity on US is not lesion specific and, on the basis of pathological correlation studies, US has poor sensitivity and specificity in detecting subtle or diffuse brain injury. ${ }^{24}$ Several studies have suggested that conventional MR imaging is more sensitive for detecting WMD than US both early after birth ${ }^{25}$ and at a corrected age of term, ${ }^{26}$ but as yet there has been no correlation with neurodevelopmental outcome. MR imaging may be helpful in the early diagnosis of PVL: diffusion weighted imaging detected PVL in a 30 week gestation infant at a time when both US and conventional MR imaging were normal. ${ }^{27}$

The American Academy of Neurology quality standards committee have suggested that, so far, there is insufficient evidence for routine MR imaging of all preterm infants with WMD on US. ${ }^{13}$ Some European studies, however, suggest that a conventional MR image at a corrected age of term can give useful prognostic information. In particular, when there is unilateral parenchymal involvement from PVL or haemorrhagic parenchymal infarction, the likelihood of subsequent hemiplegia depends on whether there is asymmetrical myelination in the posterior limb of the internal capsule on conventional MR imaging. ${ }^{28} 29$

\section{ROLE OF MR IN NEONATAL NETWORKS}

In view of its growing clinical importance, it is arguable that neonatal networks should be planned to ensure that MR imaging is available for all infants who will benefit from it. However, universal access to high quality imaging will have enormous cost, resource, and planning implications for neonatal care in the United Kingdom. It is important that the limitations and logistical problems of MR techniques are carefully considered. MR is not and will never be a bedside procedure. Because of the potential attraction of ferromagnetic objects toward the magnet core, all equipment and implants must be nonmagnetic. Infants must be stable enough to tolerate transfer to the MR facility, and require physiological monitoring during an MR study with at least one experienced neonatologist in attendance. Noise levels may be unacceptably high, and noise reduction devices such as earmuffs and/or ear plugs may be required. The quality of MR data is sensitive to movement, and sedation is often necessary. In our experience, a milk feed and $30-50 \mathrm{mg} / \mathrm{kg}$ chloral hydrate before the study is safe and effective. MR compatible ventilators and incubators are now commercially available; with careful consideration of the infant's wellbeing, many groups have successfully performed MR studies in infants receiving full intensive care. ${ }^{30} 31$

Access to a suitable MR facility is just the start; standard sequences for the adult brain are not appropriate for imaging infants and sequences need to be adapted for neonatal brain imaging to accommodate the changes in $\mathrm{Tl}$ and $\mathrm{T} 2$ as physiological myelination and growth take place. MR imaging must become more infant friendly and imaging strategies developed to provide maximum information in minimum time. MR techniques must be standardised in terms of sequence parameter, region of brain evaluated, timing of evaluation, and reference to robust control data. Improved magnet technology is needed that will allow easy placement of MR systems adjacent to neonatal intensive care units. MR scans need to be interpreted by a radiologist who has experience of neonatal brain imaging because of unusual and idiosyncratic signal changes that occur with normal maturation and growth. Results of imaging studies must be available immediately for viewing by all involved specialties. With the advent of filmless radiology, it may be possible to distribute MR images throughout a network or between network centres using internet technology such as Digital Imaging and Communications in Medicine (DICOM) and Picture Archiving and Communication Systems (PACS). ${ }^{32}$ DICOM and PACS applications have recently become commercially available. Combined with appropriate software, these applications will allow images to be accessed through the internet and MR images reported from afar.

\section{MR RESEARCH: PAST, PRESENT, AND FUTURE DIRECTIONS}

MR has an increasingly important role in research. As we aim for the possibility of perinatal brain repair, MR will be able to improve our understanding of the response of the developing brain to injury, aid the development of new neuroprotective strategies, and monitor and assess intervention and prognosis. MR research is at the crossroads between medicine, physics, chemistry, and computer science; groups with strong interdisciplinary relations will be essential to take this goal forward.

MR spectroscopy and chemical shift imaging

MR spectroscopy can provide early supplementary prognostic information in NE (fig 3) and aids diagnosis of metabolic disorders. However, MR systems capable of multinuclear MR spectroscopy are usually present only in research settings where its main role is investigating fundamental mechanisms of injury. Only through such detailed understanding of mechanism will advances in the care and treatment of the neonatal brain be possible. In the 1980s, experimental studies using ${ }^{31} \mathrm{P}$ and ${ }^{1} \mathrm{H}$ MR spectroscopy showed a biphasic pattern of energy failure after perinatal HI. ${ }^{33}{ }^{34}$ This remarkable finding was the cornerstone behind the realisation that neuroprotective strategies may interrupt the cascade of irreversible injury if administered within hours of a perinatal insult and was an important catalyst in the development of current hypothermia trials.

More recently MR spectroscopy has shown two further important concepts. Firstly, abnormal brain metabolism can persist for months to years in infants 


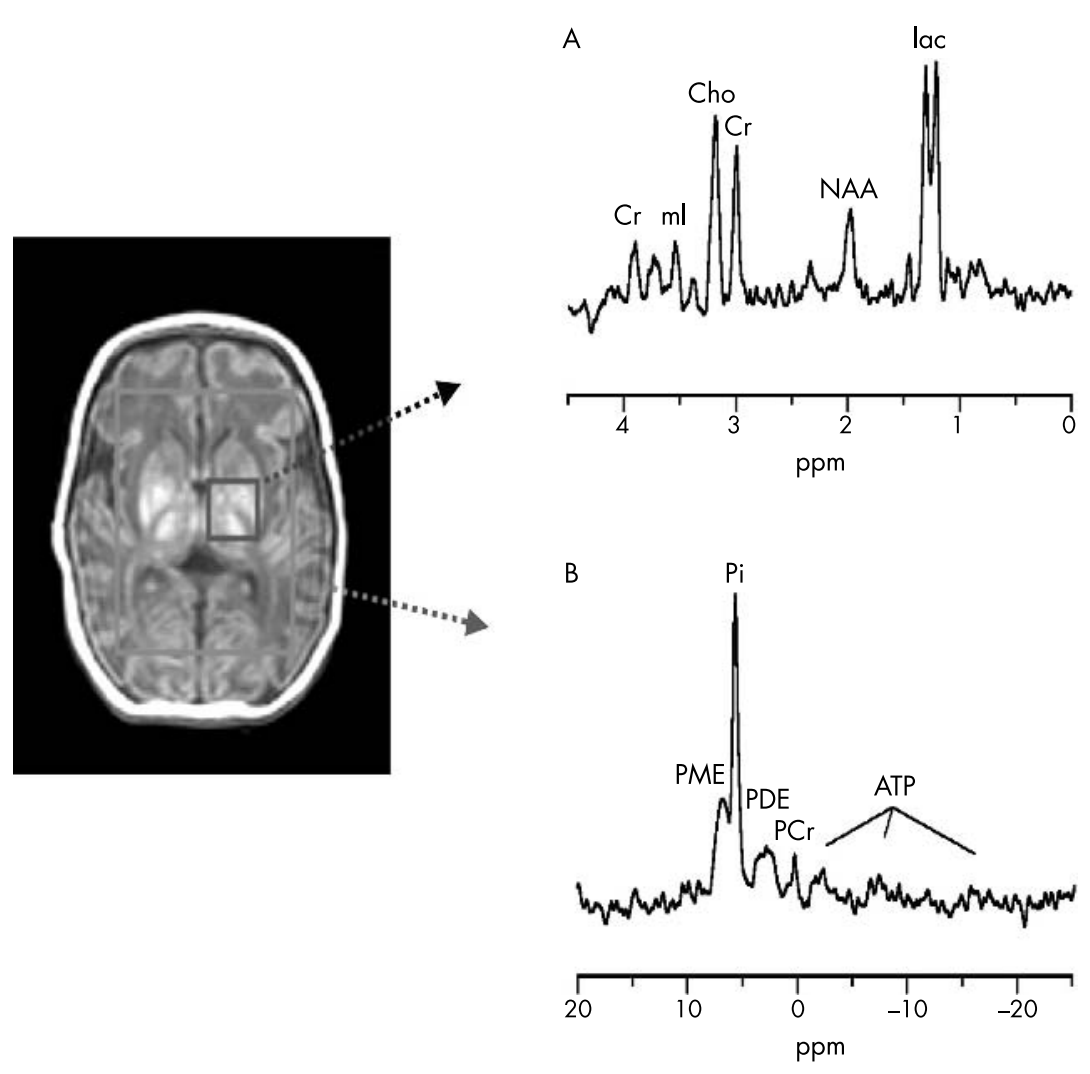

Figure 3 (A) ${ }^{1} H$ MR spectrum from left basal ganglia showing appreciably raised lactate and reduced $N$-acetyl aspartate (NAA). (B) ${ }^{31}$ P MR spectrum from the whole brain showing severe secondary energy failure with phosphocreatine (PCr) and ATP depletion and increased inorganic phosphate (Pi). The brain $\mathrm{pH}_{\mathrm{i}}$ was alkaline (7.18). PME, Phosphomonoesters; PDE, phosphodiesters; Cho, choline; $\mathrm{Cr}$, creatine; ml, myo-inositol; lac, lactate.

with a severe outcome after NE. This may indicate continuing cell death, which may be amenable to intervention for some considerable time after the insult. ${ }^{35}$ Secondly, brain $\mathrm{pH}_{\mathrm{i}}$ becomes alkaline after perinatal $\mathrm{HI}$; in vitro studies show that such intracellular alkalosis is detrimental to cell survival after HI. ${ }^{36}$ This information may help to define optimal resuscitation techniques after perinatal HI to delay rebound brain alkalosis and minimise subsequent cellular injury.

Chemical shift imaging is an extension of single voxel MR spectroscopy. Instead of obtaining spectra from one or two large voxels in the brain, this technique can obtain spectra simultaneously from numerous voxels at about $1 \mathrm{~cm}^{3}$ resolution and results in a brain metabolite concentration map. When applied to the developing brain, the technique is likely to greatly enhance our understanding of regional biochemical development and assess biochemical response to injury. Further developmental effort is needed from manufacturers to make these techniques suitable for the neonatal brain. Future applications of MR spectroscopy will also benefit from the newest generation of high strength magnets operating at 3 and $4 \mathrm{~T}$, allowing greater sensitivity in metabolite detection and increased chemical shift dispersion.

\section{Quantitative MR measurements}

Recently, a new MR era has emerged using more quantitative MR measures. Precise assessment of grey and white matter volumes has been achieved by segmentation of the imaged volume into tissue types followed by 3D rendering, and groups have begun to work on advanced segmentation techniques using atlas information to guide segmentation algorithms. This provides a powerful method of projecting atlas information into data sets of patients. ${ }^{37}$ New insights into the response of the preterm brain to injury have emerged from quantitative MR studies. Two studies from separate groups have shown that preterm infants with perinatal WMD scanned at term had a reduced volume of cortical grey matter compared with those without WMD. ${ }^{38} 39$

\section{Diffusion weighted imaging, diffusion tensor imaging, and fibre tracking}

The development of diffusion weighted imaging and diffusion tensor imaging has offered the possibility of going beyond anatomical imaging and studying tissue structure at a microscopic level in vivo. Diffusion weighted imaging measures the diffusivity of water molecules on MR imaging; an increase in signal intensity on diffusion weighted imaging is associated with the accumulation of diffusion restricted water in the intracellular space resulting from the breakdown of transmembrane pumps. White matter fibre tracts have been visualised using diffusion tensor imaging. These detailed techniques enable the assessment of the anatomical basis of the more subtle forms of preterm brain injury not detected with conventional MR imaging.

A recent study using diffusion tensor imaging in preterm infants with WMD showed that the WMD has a deleterious effect on subsequent development of white matter fibre tracts both in the area of original injury and in more distal parts of the brain containing fibres that descend from the injury such as the internal capsule. ${ }^{40}$ Such studies of neuronal connectivity hold much promise in furthering our understanding of the effect and response of injury in the preterm brain. In combination with fMRI, fibre tracking will be a powerful tool for studying neurocognitive networks and improving understanding of brain function (fig 4).

\section{Functional magnetic resonance imaging (fMRI)}

fMRI can detect regional changes in blood oxygenation associated with local metabolic activation of the brain. The technique has become a powerful tool for cognitive neuroscience research; visual, auditory, motor, and many neuropsychological tasks have been localised to specific brain regions using fMRI. Development of brain function and localisation of specific brain activity in the newborn and developing infants

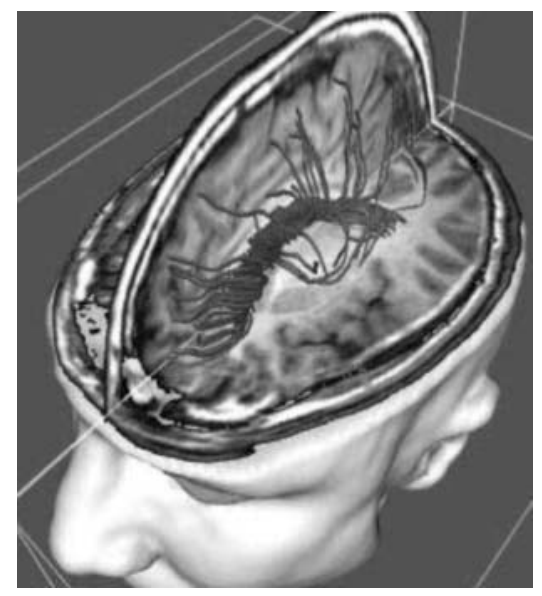

Figure 4 Fibre tracking which in the future may be possible using diffusion tensor imaging. If successful, this technique has huge potential in furthering our understanding of preterm white matter damage. 
would be of great interest although the technical problems of movement artefact and stimulus presentation are formidable. Preliminary data on neonatal fMRI in term and preterm infants are becoming available. ${ }^{41}$

\section{Magnetisation transfer imaging}

Magnetisation transfer imaging detects changes in the properties of water protons and other magnetic nuclei-for example, macromolecules-as they move from one physical state or chemical configuration to another. The most productive medical application of this technique has been the application of magnetisation transfer contrast to the study of white matter disease, notably demyelination in multiple sclerosis. This technique holds some promise in following brain myelination in the injured preterm and term infant.

\section{SUMMARY}

The rapid increase in knowledge that is being obtained using MR has been compared with the giant leap of microscopic technology from Leeuwenhoek's instrument of the 17th century to modern scanning electron microscopes. As the understanding of molecular mechanisms of disease has accelerated over the last decade, MR technology has kept pace by the development of molecular imaging aimed at exploiting specific molecules as the source of contrast. In the future, as well as observing relatively gross parameters of disease, MR will enable noninvasive exploration beyond the tissue to the cell or even molecular level. ${ }^{42}$ In the light of these developments, the full impact of MR on our understanding and treatment of the neonatal brain can only be guessed at. In the planning of future neonatal services it is important that access to MR facilities and expertise is considered to ensure that all babies at risk of brain injury will benefit from these developments.

\section{ACKNOWLEDGEMENTS}

We thank Roger Ordidge and David Gadian for helpful advice on the preparation of this article.

\section{Arch Dis Child Fetal Neonatal Ed \\ 2004;89:F193-F197.}

doi: $10.1136 /$ adc. 2003.027334

\section{Authors' affiliations}

N J Robertson, Department of Paediatrics, Faculty of Medicine, Imperial College London, Hammersmith Hospital, DuCane Road, London W12 OHS, UK

J S Wyatt, Perinatal Brain Repair Group, Department of Paediatrics and Child Health, University College London, London WC1E 6JJ, UK

Correspondence to: Dr Robertson, Perinatal Brain Repair Group, Department of Obstetrics and Gynaecology, University College London, 86-96 Chenies Mews, London WCIE 6HX, UK. n.robertson@ucl.ac.uk

\section{REFERENCES}

1 Bloch F, Hansen WW, Packard M. The nuclear induction experiment. Phys Rev 1946:70:474-85.

2 Purcell EM, Torrey HC, Pound RV. Resonance absorption by nuclear magnetic moments in a solid. Phys Rev 1946:69:37-8.

3 Doyle FH, Gore JC, Pennock JM, et al. Imaging of the brain by nuclear magnetic resonance. Lancet 1981;2:53-7.

4 Pharaoh PO, Platt MJ, Cooke T. The changing epidemiology of cerebral palsy. Arch Dis Child Fetal Neonatal Ed 1996;75:F169-73.

5 Piecuch RE, Leonard $\mathrm{CH}$, Cooper BA, et al. Outcome of extremely low birth weight infants (500 to 999 grams) over a 12-year period. Pediatrics 1997; 100:633-9.

6 Wood NS, Marlow N, Costeloe K, et al. Neurologic and developmental disability after extremely preetrm birth. N Engl J Med 2001;343:378-84.

7 Rutherford MA, Pennock J, Schwieso J, et al. Hypoxic-ischaemic encephalopathy: early MRI findings in relation to outcome. Arch Dis Child Fetal Neonatal Ed 1996;75:F145-51.

8 Rutherford MA, Pennock JM, Counsell SJ, et al Abnormal magnetic resonance signal in the internal capsule predicts poor neurodevelopmental outcome in infants with hypoxic-ischaemic encephalopathy. Pediatrics 1998; 102:323-8.

9 Cowan FM. Outcome after intrapartum asphyxia in term infants. Semin Neonatol 2000:5:127-40.

10 Patel S, Barkovich AJ. Analysis and classification of cerebellar malformations. AJNR Am J Neuroradiol 2002;23:1074-87.

11 Rutherford MA, Pennock JM, Dubowitz LMS Cranial ultrasound and magnetic resonance imaging in hypoxic-ischaemic encephalopathy: a comparison with outcome. Dev Med Child Neurol 1994;36:813-25.

12 Archer LN, Levene MI, Evans DH. Cerebral artery Doppler ultrasonography for prediction of outcome after perinatal asphyxia. Lancet 1986;15:1116-18.

13 Ment LR, Bada HS, Barnes P, et al. Practice parameter: neuroimaging of the neonate. Report of the Quality Standards Subcommittee of the American Academy of Neurology and the Practice Committee of the Child Neurology Society. Neurology 2002;58:1726-38.

14 Barkovich AJ, Westmark KD, Bedi HS, et al. Proton spectroscopy and diffusion imaging on the first day of life after perinatal asphyxia: preliminary report. AJNR Am J Neuroradio $2001 ; 22: 1786-94$.

15 Warach S, Chien D, Li W, et al. Fast magnetic resonance diffusion weighted imaging of acute human stroke. Neurology 1992;42:1717-23.

16 Moseley ME, Cohen Y, Mintorovitch J, et al. Early detection of regional cerebral ischaemia in cats: comparison of diffusion and T2 weighted MRI and spectroscopy. Magn Reson Med 1990; 14:330-46.

17 Toet MC, Hellstrom-Westas L, Groenendaal F, et al Amplitude integrated EEG 3 and 6 hours after birth in full term neonates with hypoxic-ischaemic encephalopathy. Arch Dis Child Fetal Neonatal Ed 1999;81:F19-23.

18 Pressler RM, Boylan GB, Morton M, et al. Early serial EEG in hypoxic ischaemic encephalopathy. Clin Neurophys 2001;112:31-7.

19 Mercuri E, Cowan FM, Gupte G, et al. Prothrombotic disorders and abnormal neurodevelopmental outcome in infants with neonatal cerebral infarction. Pediatrics 2001; 107:1400-4.

20 Mercuri E, Cowan FM, Rutherford $M$, et al. Ischaemic and haemorrhagic brain lesions in newborns with seizures and normal Apga scores. Arch Dis Child Fetal Neonatal Ed 1995;73:F67-74.

21 Cowan FM, Pennock J, Hanrahan D, et al. Early detection of cerebral infarction and hypoxicischaemic encephalopathy in neonates using diffusion-weighted MRI. Neuropediatrics 1994;25:172-5.

22 Mercuri E, Rutherford MA, Cowan FM, et al. Early prognostic indicators of outcome in infants with neonatal cerebral infarction: a clinical, EEG and MRI study. Pediatrics 1999;103:39-46.

23 Dammann O, Leviton A. The role of perinatal brain damage in developing disabilities: an epidemiological perspective. Ment Retard Dev Disabil Res Rev 1997;3:263-8.

24 Paneth N, Rudelli R, Monte W, et al. White matter necrosis in very low birthweight infants: neuropathologic and ultrasonographic findings in infants surviving six days or longer. J Pediatr 1990;1 16:975-84.

25 Maalouf EF, Duggan PJ, Counsell SJ, et al. Comparison of findings on cranial ultrasound and MRI in preterm infants. Pediatrics 2001;107:719-27.

26 Maalouf EF, Duggan PJ, Rutherford MA, et al. Magnetic resonance imaging in a cohort of extremely preterm infants. J Pediatr 1999:135:351-7.

27 Inder T, Huppi PS, Zientara GP, et al. Early detection of periventricular leukomalacia by diffusion-weighted magnetic resonance imaging techniques. J Pediatr 1999:134:631-4.

28 Roelants-van Rijn AM, Groenendaal F, Beek FJ, et al. Parenchymal brain injury in the preterm infant: comparison of cranial ultrasound, MRI and neurodevelopmental outcome. Neuropediatrics 2001:32:80-9.

29 de Vries LS, Groenendaal F, Van Haastert IC, et al. Asymmetrical myelination of the posterior limb of the internal capsule in infants with periventricular haemorrhagic infarction: an early predictor of hemiplegia. Neuropediatrics 1999;30:1-6.

30 Groenendaal F, Leusink $C$, Nijenhuis $M$, et al. Neonatal life support during MR imaging. J Med Eng Technol 2002;26:71-4.

31 Battin M, Maalouf EF, Counsell SJ, et al. Physiological stability of preterm inafnst during MR imaging. Early Hum Dev 1998:52:101-10.

32 Ernst R, Le VT, Kawashima A, et al. A picture archiving and communications system featuring multiple monitors using windows 98. J Digit Imaging 1999; 12:106-8.

33 Lorek AY, Takei EB, Cady JS, et al. Delayed ('secondary') cerebral energy failure following acute hypoxia-ischaemia in the newborn piglet: continuous 48 -hour studies by ${ }^{31} \mathrm{P}$ MRS. Pediatr Res 1994;36:699-706.

34 Penrice J, Lorek A, Cady EB, et al. Proton magnetic resonance spectroscopy of the brain during acute hypoxia-ischemia and delayed cerebral energy failure in the newborn piglet. Pediatr Res 1997:41:795-802.

35 Robertson NJ, Cox IJ, Cowan FM, et al. Cerebral intracellular lactic alkalosis persisting months after neonatal encephalopathy measured by magnetic resonance spectroscopy. Pediatr Res 1999:46:287-96

36 Robertson NJ, Cowan FM, Cox IJ, et al. Brain alkaline intracellular $\mathrm{pH}$ and adverse neurodevelopmental outcome after neonatal encephalopathy. Ann Neurol 2002;52:732-42.

37 Huppi PS, Warfield S, Kikinis R, et al. Quantitative MRI of brain development in premature and mature newborns. Ann Neurol 1998;43:224-35.

38 Inder T, Huppi P, Warfield S, et al Periventricular white matter damage in the preterm infant is associated with a reduction in cerebral cortical grey matter at term. Ann Neurol 1999:46:755-60

39 Ajayi-Obe M, Saeed N, Cowan FM, et al Reduced development of cerebral cortex in extremely preterm infants. Lancet 2000;356: 1162-3.

40 Huppi PS, Murphy B, Maier SE, et al Microstructural brain development after perinatal cerebral white matter injury assessed by diffusion fensor magnetic resonance imaging. Pediatrics 2001;107:455-60.

41 Peterson BS, Vohr B, Kane MJ, et al. A functional magnetic resonance study of language processing and its cognitive correlates in prematurely born children. Pediatrics 2002;110:1153-62.

42 Weissleder R. Molecular imaging: exploring the next frontier. Radiology 1999;212:638-44. 